\title{
Planktonic and Non-Planktonic Rotifers in Two Environments of the Upper Paraná River Floodplain, State of Mato Grosso do Sul, Brazil
}

\author{
Cláudia C. Bonecker*, Fábio A. Lansac-Tôha and Dayane C. Rossa \\ State University of Maringá/DBI/Nupelia/Postgraduate Course in Continental Aquatic Environments, Av. Colombo, \\ 5790, 87020-900, Maringá, PR, Brazil
}

\begin{abstract}
Investigations were carried out for the composition and occurrence of rotifer taxa of different forms and sizes during two distinct hydrological periods, in two environments (river and lake). Two sampling stations were established in open water of each environments. Surface samples were taken daily for 14 days. The faunistic similarity between the environments was established using Jaccard's index. One hundred and fourty three taxa were identified. The largest number of taxa was recorded in the river. Faunistic similarity between these environments was greater during high water. The planktonic taxa predominated and the difference for the non-planktonic ones was not marked. Non-planktonic taxa predominated in the river, while both types were numerous in the lake. We observed a larger number of smaller-sized taxa in both environments ( $\geq 200 \mu \mathrm{m}$ to $600 \mu \mathrm{m}$ in length). This was related to the shallowest depths and to current velocity, which moved benthic and periphytic taxa from the bottom and littoral zone into the water column.
\end{abstract}

Key Words: Rotifers, Size classes, Habitat, Floodplain, Brazil

\section{INTRODUCTION}

Variations in fluviometric levels on floodplains influence abiotic changes in the environments of these ecosystems. This fluctuation is associated with environmental heterogeneity and, together with interspecific and intraspecific relationships within the aquatic communities, is responsible for the high biodiversity in the floodplain.

The relatively high diversities of rotifer communities of floodplains have been described by several authors in South America (Hardy, 1980; Robertson \& Hardy, 1984; Vásquez, 1984; Paggi \& José de Paggi, 1990; Bozelli, 1994; Bonecker \& Lansac-Tôha, 1996; Espíndola et al., 1996). Most of the studies discuss the variations of the rotifer composition over broad time scales. However, their composition may also vary over short intervals (Twombly \& Lewis Jr., 1987; Vásquez \& Rey, 1989; Hillman \& Shiel, 1991; Tan \& Shiel, 1993). Short-term variations may be related to (i) variation in environmental conditions, favoring one species to the detriment of another, (ii) the biotic relationships, competition, and predation, as well as (iii) the interactions of these factors.

This investigation treats the composition and occurrence of rotifer taxa of different forms and sizes in short period of time (14 days) during two distinct hydrological periods (high water and low water), in two environments of the Upper Paraná River floodplain, state of Mato Grosso do Sul.

\section{STUDY AREA}

The Baía River $\left(22^{0} 43^{\prime} \mathrm{S}\right.$ and $\left.53^{\circ} 17^{\prime} \mathrm{W}\right)$ is formed by a large number of lakes along its course. The low velocity of its current and the slight slope of its bed produce the characteristics of a semi-lotic environment. Along the stretch sampled, a number of grasses, chiefly Panicum prionitis Ness (Souza et al., 1997) are present along the low banks.

\footnotetext{
* Author for correspondence
} 
Guaraná Lake (22o43'S and 53o18'W) is situated on the right bank of the Lower Baía River and communicates with the river by means of a permanent channel. Guaraná Lake can be described as a "várzea" lake and has no defined limits, since it grades subtly into flooded areas. Aquatic macrophytes, chiefly Eichhornia azurea (Schwartz) Kunth, are predominant in the channel and in the littoral region.

The waters of both river and lake are warm (15$30^{\circ} \mathrm{C}$ ), with acid to neutral $\mathrm{pH}$ (5.2-7.1), low electrical conductivity $(17-68 \mu \mathrm{S} / \mathrm{cm})$ and low dissolved oxygen concentrations (3.7-116.2\% sat.) (Thomaz et al., 1991, 1992).

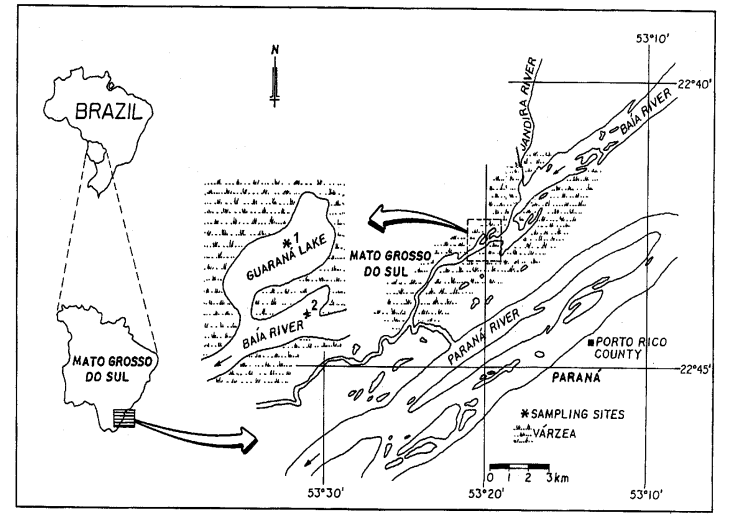

Fig. 1. The study area and sampling stations.

\section{MATERIAL AND METHODS}

Two sampling stations were established in open water, one in the Baía River and another in Guaraná Lake. Samples were taken daily for 14 days, at the surface, during low water (August/93) and high water (March/94). Rotifers were collected with a motorized pump from open water, using a plankton net of $70 \mu \mathrm{m}$ mesh size.

Rotifers (ind $/ \mathrm{m}^{3}$ ) were quantified by counting subsamples in a Sedgwick-Rafter cell; at least 200 individuals of each sample were counted. Identification was based on Koste (1978), Koste \& Robertson (1983), José de Paggi (1989), and Segers (1995) methods.
The taxa were classified into size groups: (i) class 1 (c1), individuals up to $200 \mu \mathrm{m}$ long, (ii) class 2 (c2), $\geq 200 \mu \mathrm{m}$ to $<600 \mu \mathrm{m}$, (iii) class 3 (c3), $\geq 600 \mu \mathrm{m}$ to $<1000 \mu \mathrm{m}$, and (iv) class 4 (c4), $\geq 1000 \mu \mathrm{m}$. Each taxon was also classified according to its preferred habitat, as (i) planktonic or (ii) non-planktonic (periphytic and benthic taxa). The sizes of each taxa and their preferred habitat were described according to Koste (1978) and Segers (1995).

Faunistic similarity in each environment was established using Jaccard's index (Krebs, 1989), according to the hydrological cycle (high water and low water).

\section{RESULTS}

\section{Hydrological cycle}

The daily data for depth of the Baía River and Guaraná Lake showed two distinct periods: low water, with depth between $1,4 \mathrm{~m}$ and $2,4 \mathrm{~m}$ (August/93) and high water, with depth between 2,8 $\mathrm{m}$ and 3,4 $\mathrm{m}$ (March/94) (Fig. 2).

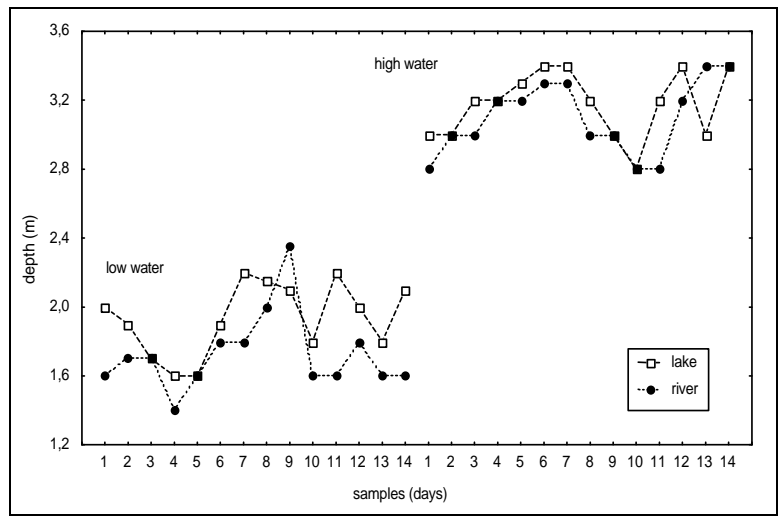

Fig. 2. Daily variations of depths (m) in the Baía River and Guaraná Lake (low water, August/93; high water, March/94).

\section{Rotifer composition}

We identified 143 taxa of rotifers belonging to 21 families (Table 1). Ascomorpha klementi, Elosa sp., Lecane furcata, $L$. simonneae, $L$. lunaris f. constricta, Lepadella quadricarinata, L. brasiliensis, Mytilinia bicarinata, Notholca sp., Pompholyx cf. triloba, Proales cf. decipiens, Testudinella tridentata, Trichocerca agnatha, $T$. 
inermis, T. mucosa bicristata, T. rattus minor, $T$. jeningsi, T. ruttneri, and T. sulcata are new records for the Upper Paraná River floodplain. Except for $L$. furcata and T. tridentata, which were recorded on the Middle Paraná River floodplain (José de Paggi, 1996), Trichocerca mucosa bicristata, on the Upper Paraná River floodplain (Sendacz, 1995), and T. mucronata, which was found in environments of the Middle Paraná River and the Upper Paraná floodplain, the other new records represent new occurrences for the entire Paraná River floodplain, including Brazil and Argentina.

One hundred and nineteen taxa were recorded in the river and 112 in the lake. However, there was little difference in rotifer composition between the two environments ( 7 taxa).

A high number of planktonic taxa was recorded, but there was no marked difference between habitats for the non-planktonic taxa (4 taxa) (Table 1). However, there were difference in the environments (Table 2). Many non-planktonic taxa were found in the river, while both planktonic and non-planktonic taxa were numerous in the lake.

Small-sized taxa $(\mathrm{c} 1=$ up to $200 \mu \mathrm{m}, \mathrm{c} 2=\geq 200$ to $600 \mu \mathrm{m}$ ) were most numerous (Table 1). In both environments, there was a high number of taxa $\geq 200 \mu \mathrm{m}$ and $600 \mu \mathrm{m}$ (Table 2).

Table 2. Number of taxa recorded in each environment, during high water (HW) and low water (LW), according to size class (c1= up to $200 \mu \mathrm{m}$; $\mathrm{c} 2=\geq 200$ to $600 \mu \mathrm{m} ; \mathrm{c} 3=\geq$ 600 to $<1000 \mu \mathrm{m}$; and $\mathrm{c} 4=\geq 1000 \mu \mathrm{m})$ and preferred habitat (npk=non-planktonic, pk=planktonic).

\begin{tabular}{|c|c|c|c|c|c|c|}
\hline Environment & \multicolumn{3}{|c|}{ Lake } & \multicolumn{3}{|c|}{ River } \\
\hline & $\mathbf{N}$ & HW & LW & $\mathbf{N}$ & HW & LW \\
\hline Number of taxa & 112 & 99 & 78 & 119 & 116 & 63 \\
\hline Size $\quad$ c1 & 47 & 39 & 31 & 52 & 49 & 24 \\
\hline \multirow[t]{3}{*}{ Classes } & 55 & 49 & 38 & 56 & 58 & 31 \\
\hline & 06 & 06 & 05 & 07 & 05 & 05 \\
\hline & 04 & 05 & 04 & 04 & 04 & 03 \\
\hline \multirow[t]{2}{*}{ Habitat } & 54 & 53 & 37 & 62 & 64 & 26 \\
\hline & 58 & 46 & 41 & 57 & 52 & 37 \\
\hline
\end{tabular}

Increases in water depth influenced rotifer composition in both river and lake. More taxa were recorded during high water, mainly in the river. During high water, we observed a high number of taxa belonging to size class c2 ( $\geq$ $200 \mu \mathrm{m}$ to $600 \mu \mathrm{m}$ ), and many non-planktonic taxa.

High differences in rotifer composition were recorded in the low water period, with more taxa found in the lake. During high water, the number of taxa was similar (Fig. 3). Jaccard's index showed that faunistic similarity was greater during high water (0.69) than low water (0.61).

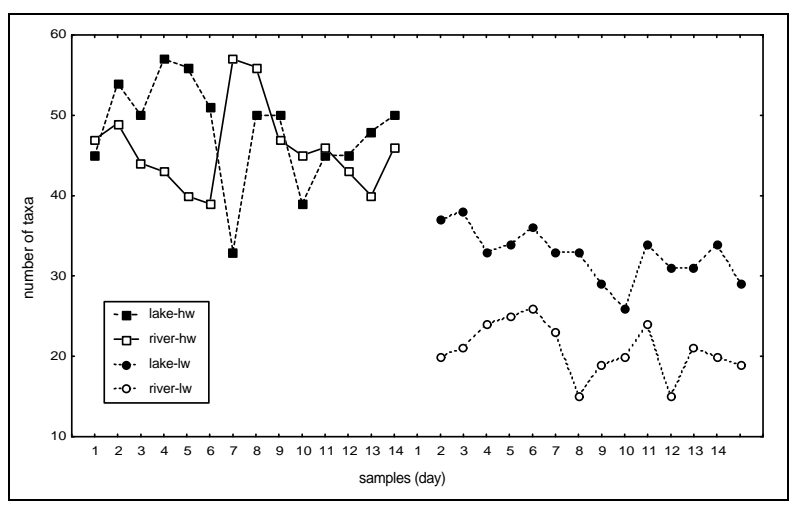

Fig. 3. Daily variations in number of taxa during high water (hw) and low water (lw).

\section{DISCUSSION}

The 144 taxa identified in this study indicated the high diversity of rotifers in the floodplain, probably because of environmental heterogeneity. The relationship between diversity and habitat heterogeneity was also observed by José de Paggi (1996) in floodplain environments of the Middle Paraná River.

Rocha et al. (1995) showed that 519 rotifer taxa have been recorded in different environments in South America. Lansac-Tôha et al. (1997) and Serafim (1997) identified a total of 198 taxa in the Upper Paraná floodplain, which our results have increased to 218 .

A large number of taxa of the families Brachionidae, Lecanidae, and Trichocercidae have also been observed in other environments of this floodplain (Lansac-Tôha et al., 1997; Rossa, 1997; Serafim, 1997), in South American floodplains (Robertson \& Hardy, 1984; Bozelli, 1992; López \& Ochoa, 1995), and in Africa (Segers et al., 1993). 
Table 1. Composition of rotifers occurring in the lake $(\mathrm{L})$ and the river $(\mathrm{R})$, with date for: $\mathrm{t}=$ size, cl=size classes (c1= up to $200 \mu \mathrm{m} ; \mathrm{c} 2=\geq 200$ to $600 \mu \mathrm{m} ; \mathrm{c} 3=\geq 600$ to $<1000 \mu \mathrm{m}$; and $\mathrm{c} 4=\geq 1000 \mu \mathrm{m}$ ), h=preferred habitat (npk=non-planktonic, $\mathrm{pk}=$ planktonic); and $\mathrm{b}=$ bibliographic reference to indicate the size class and preferred habitat (1=Koste, 1978; 2=Koste \& Shiel, 1986; 3=Segers, 1995).

\begin{tabular}{|c|c|c|c|c|c|c|c|}
\hline \multirow[t]{2}{*}{ Families } & \multirow[t]{2}{*}{ Taxa } & \multicolumn{2}{|c|}{ Occurrence } & \multirow[t]{2}{*}{$\mathbf{t}(\mu \mathbf{m})$} & \multirow[t]{2}{*}{ cl } & \multirow[t]{2}{*}{$\mathbf{h}$} & \multirow[t]{2}{*}{$\mathbf{b}$} \\
\hline & & $\mathbf{L}$ & $\mathbf{R}$ & & & & \\
\hline \multirow[t]{2}{*}{ Philodinidae } & Dissotrocha aculeata (Ehrenberg, 1832) & $\mathrm{x}$ & $\mathrm{x}$ & 500 & $\mathrm{c} 2$ & $\mathrm{npk}$ & 2 \\
\hline & Others Bdelloidea & $\mathrm{x}$ & $\mathrm{x}$ & & & & \\
\hline \multirow[t]{19}{*}{ Brachionidae } & Anueropsis fissa (Gosse, 1851) & $\mathrm{x}$ & & 120 & $\mathrm{c} 1$ & $\mathrm{pk}$ & 1 \\
\hline & Brachionus angularis Gosse, 1851 & $\mathrm{x}$ & $\mathrm{x}$ & 210 & $\mathrm{c} 2$ & pk & 1 \\
\hline & B. calyciflorus Pallas, 1766 & $\mathrm{x}$ & $\mathrm{x}$ & 600 & $\mathrm{c} 3$ & pk & 1 \\
\hline & B. caudatus Barrois \& Daday 1884 & $\mathrm{x}$ & $\mathrm{x}$ & 270 & $\mathrm{c} 2$ & npk & 1 \\
\hline & B. c. f personatus Ahlstrom, 1940 & & $\mathrm{x}$ & 270 & $\mathrm{c} 2$ & $\mathrm{pk}$ & 1 \\
\hline & B. dolabratus Harring, 1915 & $\mathrm{x}$ & $\mathrm{x}$ & 160 & $\mathrm{c} 1$ & npk & 1 \\
\hline & B. mirus (Koste, 1972) & $\mathrm{x}$ & $\mathrm{x}$ & 280 & $\mathrm{c} 2$ & $\mathrm{pk}$ & 1 \\
\hline & B. quadridentatus Hermann, 1783 & $\mathrm{x}$ & $\mathrm{x}$ & 415 & $\mathrm{c} 2$ & $\mathrm{pk}$ & 1 \\
\hline & B. falcatus Zacharias, 1898 & $\mathrm{x}$ & $\mathrm{x}$ & 430 & $\mathrm{c} 2$ & $\mathrm{pk}$ & 1 \\
\hline & Keratella americana Carlin, 1943 & $\mathrm{x}$ & $\mathrm{x}$ & 320 & $\mathrm{c} 2$ & $\mathrm{pk}$ & 1 \\
\hline & K. cochlearis Gosse, 1851 & $\mathrm{x}$ & $\mathrm{x}$ & 320 & $\mathrm{c} 2$ & $\mathrm{pk}$ & 1 \\
\hline & K. tropica (Apstein, 1907) & $\mathrm{x}$ & $\mathrm{x}$ & 300 & $\mathrm{c} 2$ & $\mathrm{pk}$ & 1 \\
\hline & K. lenzi (Hauer, 1953) & $\mathrm{x}$ & $\mathrm{x}$ & 160 & $\mathrm{c} 1$ & $\mathrm{pk}$ & 1 \\
\hline & Notholca sp. & $\mathrm{x}$ & $\mathrm{x}$ & & & & \\
\hline & Platyias leloupi (Gillard, 1957) & $\mathrm{x}$ & & 410 & $\mathrm{c} 2$ & $\mathrm{pk}$ & 1 \\
\hline & P. quadricornis quadricornis (Ehrenberg, 1832) & $\mathrm{x}$ & $\mathrm{x}$ & 576 & $\mathrm{c} 2$ & $\mathrm{pk}$ & 1 \\
\hline & P. q. brevispinus Daday, 1905 & $\mathrm{x}$ & $\mathrm{x}$ & 410 & $\mathrm{c} 2$ & $\mathrm{pk}$ & 1 \\
\hline & Plationus patulus (O. F. Müller, 1786) & $\mathrm{x}$ & $\mathrm{x}$ & 265 & $\mathrm{c} 2$ & npk & 1 \\
\hline & P. p. macracanthus (Daday, 1905) & $\mathrm{x}$ & $\mathrm{x}$ & 300 & $\mathrm{c} 2$ & npk & 1 \\
\hline \multirow[t]{24}{*}{ Trichocercidae } & Trichocerca agnatha Wulf, 1939 & $\mathrm{x}$ & $\mathrm{x}$ & 230 & $\mathrm{c} 2$ & $\mathrm{pk}$ & 1 \\
\hline & T. bicristata Gosse, 1886 & $\mathrm{x}$ & $\mathrm{x}$ & 660 & $\mathrm{c} 3$ & $\mathrm{pk}$ & 1 \\
\hline & T. bicristata var. mисоsa (Stokes, 1896) & & $\mathrm{x}$ & 350 & $\mathrm{c} 2$ & pk & 1 \\
\hline & T. bidens (Lucks, 1912) & & $\mathrm{x}$ & 240 & $\mathrm{c} 2$ & $\mathrm{pk}$ & 1 \\
\hline & T. c. chattoni De Beauchamp, 1907 & $\mathrm{x}$ & $\mathrm{x}$ & 448 & $\mathrm{c} 2$ & $\mathrm{pk}$ & 1 \\
\hline & T. capucina Wierzejski \& Zacharias, 1893 & $\mathrm{x}$ & $\mathrm{x}$ & 430 & $\mathrm{c} 2$ & $\mathrm{pk}$ & 1 \\
\hline & T. cylindrica (Imhof, 1891) & $\mathrm{x}$ & $\mathrm{x}$ & 795 & $\mathrm{c} 3$ & $\mathrm{pk}$ & 1 \\
\hline & T. collaris Carlin, 1939 & $\mathrm{x}$ & $\mathrm{x}$ & 309 & $\mathrm{c} 2$ & $\mathrm{pk}$ & 1 \\
\hline & T. elongata (Gosse, 1886) & $\mathrm{x}$ & $\mathrm{x}$ & 810 & $\mathrm{c} 3$ & $\mathrm{pk}$ & 1 \\
\hline & T. flagellata Hauer, 1937 & $\mathrm{x}$ & & 247 & $\mathrm{c} 2$ & npk & 1 \\
\hline & T. gracilis $($ Tessin, 1890$)$ & $\mathrm{x}$ & & 227 & $\mathrm{c} 2$ & $\mathrm{npk}$ & 1 \\
\hline & T. heterodactyla (Tschugunoff, 1921) & $\mathrm{x}$ & $\mathrm{x}$ & 308 & $\mathrm{c} 2$ & $\mathrm{pk}$ & 1 \\
\hline & T. inermis (Gosse, 1887) & $\mathrm{X}$ & $\mathrm{x}$ & 135 & $\mathrm{c} 1$ & $\mathrm{pk}$ & 1 \\
\hline & T. insiginis (Herrick, 1885) & $\mathrm{x}$ & $\mathrm{x}$ & 376 & $\mathrm{c} 2$ & $\mathrm{pk}$ & 1 \\
\hline & T. jenningsi Voigt, 1957 & & $\mathrm{x}$ & 408 & $\mathrm{c} 2$ & $\mathrm{pk}$ & 1 \\
\hline & T. porcellus Weber, 1898 & $\mathrm{x}$ & $\mathrm{x}$ & 110 & $\mathrm{c} 1$ & $\mathrm{pk}$ & 1 \\
\hline & T. pusilla (Lauterborn, 1898) & $\mathrm{x}$ & $\mathrm{x}$ & 175 & $\mathrm{c} 1$ & $\mathrm{pk}$ & 1 \\
\hline & T. rattus (O. F. Müller, 1776) & $\mathrm{x}$ & $\mathrm{x}$ & 320 & $\mathrm{c} 2$ & pk & 1 \\
\hline & T. rattus f. minor (Fadeew, 1925) & $\mathrm{x}$ & $\mathrm{x}$ & 270 & $\mathrm{c} 2$ & $\mathrm{pk}$ & 1 \\
\hline & T. rosea $($ Stenroos, 1898$)$ & $\mathrm{x}$ & $\mathrm{x}$ & 336 & $\mathrm{c} 2$ & $\mathrm{pk}$ & 1 \\
\hline & T. rousseleti (Voigt, 1902) & $\mathrm{x}$ & $\mathrm{x}$ & 145 & $\mathrm{c} 1$ & $\mathrm{pk}$ & 1 \\
\hline & T. ruttneri (Donner, 1953) & $\mathrm{x}$ & $\mathrm{X}$ & 189 & $\mathrm{c} 1$ & $\mathrm{pk}$ & 1 \\
\hline & T. scipio Pejler, 1962 & $\mathrm{x}$ & $\mathrm{x}$ & 408 & $\mathrm{c} 2$ & $\mathrm{pk}$ & 1 \\
\hline & T. similis (Wierzejski, 1893) & $\mathrm{x}$ & $\mathrm{x}$ & 300 & $\mathrm{c} 2$ & $\mathrm{pk}$ & 1 \\
\hline
\end{tabular}




\begin{tabular}{|c|c|c|c|c|c|c|c|}
\hline \multirow[t]{2}{*}{ Families } & \multirow{2}{*}{ Taxa } & \multicolumn{2}{|c|}{ Occurrence } & \multirow{2}{*}{$\mathbf{t}(\mu \mathbf{m})$} & \multirow{2}{*}{ cl } & \multirow[t]{2}{*}{$\mathbf{h}$} & \multirow[t]{2}{*}{ b } \\
\hline & & $\mathbf{L}$ & $\mathbf{R}$ & & & & \\
\hline & T. stylata (Gosse, 1851) & $\mathrm{x}$ & $\mathrm{x}$ & 230 & $\mathrm{c} 2$ & $\mathrm{pk}$ & 1 \\
\hline & T. sulcata (Jennings, 1894) & $\mathrm{x}$ & & 80 & $\mathrm{c} 1$ & npk & 1 \\
\hline & T. stylata (Gosse, 1851$)$ & $\mathrm{x}$ & $\mathrm{x}$ & 230 & $\mathrm{c} 2$ & $\mathrm{pk}$ & 1 \\
\hline & T. sulcata (Jennings, 1894) & $\mathrm{x}$ & & 80 & $\mathrm{c} 1$ & $\mathrm{npk}$ & 1 \\
\hline Epiphanidae & Epiphanes clavulata (Ehrenberg, 1832) & & $\mathrm{x}$ & 600 & $\mathrm{c} 3$ & npk & 1 \\
\hline \multirow{2}{*}{ Trochosphaeridae } & Trochocosphaera aequatorialis (Semper, 1872) & & $\mathrm{x}$ & 1100 & $\mathrm{c} 4$ & $\mathrm{pk}$ & 1 \\
\hline & Horäella thomansoni Koste, 1973 & $\mathrm{x}$ & & 140 & $\mathrm{c} 1$ & $\mathrm{pk}$ & 1 \\
\hline \multirow[t]{25}{*}{ Lecanidae } & Lecane aculeata (Jakubski, 1912) & $\mathrm{x}$ & $\mathrm{x}$ & 67 & $\mathrm{c} 1$ & $\mathrm{npk}$ & 3 \\
\hline & L. amazonica (Murray, 1913) & & $\mathrm{x}$ & 106 & $\mathrm{c} 1$ & $\mathrm{npk}$ & 3 \\
\hline & L. bulla (Gosse, 1851) & $\mathrm{x}$ & $\mathrm{x}$ & 133 & $\mathrm{c} 1$ & $\mathrm{npk}$ & 3 \\
\hline & L. cornuta (O. F. Müller, 1776) & & $\mathrm{x}$ & 128 & $\mathrm{c} 1$ & $\mathrm{npk}$ & 3 \\
\hline & L. closterocerca Schmarda, 1859 & $\mathrm{x}$ & $\mathrm{x}$ & 85 & $\mathrm{c} 1$ & $\mathrm{npk}$ & 3 \\
\hline & L. curvicornis (Murray, 1913) & $\mathrm{x}$ & $\mathrm{x}$ & 120 & $\mathrm{c} 1$ & npk & 3 \\
\hline & L. elsa Hauer, 1931 & $\mathrm{x}$ & $\mathrm{x}$ & 140 & $\mathrm{c} 1$ & $\mathrm{npk}$ & 3 \\
\hline & L. furcata (Murray, 1913) & $\mathrm{x}$ & $\mathrm{x}$ & 76 & $\mathrm{c} 1$ & $\mathrm{npk}$ & 3 \\
\hline & L. halycista Harring \& Myers, 1926 & & $\mathrm{x}$ & 92 & $\mathrm{c} 1$ & npk & 3 \\
\hline & L. hamata (Stokes, 1896) & $\mathrm{x}$ & $\mathrm{x}$ & 88 & $\mathrm{c} 1$ & npk & 3 \\
\hline & L. hornemani (Ehrenberg, 1834) & $\mathrm{x}$ & $\mathrm{x}$ & 110 & $\mathrm{c} 1$ & $\mathrm{npk}$ & 3 \\
\hline & L. inopinata Harring \& Myers, 1926 & & $\mathrm{x}$ & 75 & $\mathrm{c} 1$ & $\mathrm{npk}$ & 3 \\
\hline & L. leontina (Turner, 1892) & $\mathrm{x}$ & $\mathrm{x}$ & 170 & $\mathrm{c} 1$ & npk & 3 \\
\hline & L. ludiwigii (Eckstein, 1883) & $\mathrm{x}$ & $\mathrm{x}$ & 140 & $\mathrm{c} 1$ & $\mathrm{npk}$ & 3 \\
\hline & L. luna (O. F. Müller, 1776) & & $\mathrm{x}$ & 162 & $\mathrm{c} 1$ & $\mathrm{npk}$ & 3 \\
\hline & L. lunaris (Ehrenberg, 1832) & $\mathrm{x}$ & $\mathrm{x}$ & 115 & $\mathrm{c} 1$ & npk & 3 \\
\hline & L. l. f constricta (Murray, 1913) & $\mathrm{x}$ & $\mathrm{x}$ & 129 & $\mathrm{c} 1$ & $\mathrm{npk}$ & 1 \\
\hline & L. monostyla (Daday, 1897) & $\mathrm{x}$ & $\mathrm{x}$ & 58 & $\mathrm{c} 1$ & $\mathrm{npk}$ & 3 \\
\hline & L. proiecta Hauer, 1956 & $\mathrm{x}$ & $\mathrm{x}$ & 113 & $\mathrm{c} 1$ & npk & 3 \\
\hline & L. signifera (Jennings, 1894) & $\mathrm{x}$ & $\mathrm{x}$ & 190 & $\mathrm{c} 1$ & $\mathrm{npk}$ & 3 \\
\hline & L. simmoneae Segers, 1993 & $\mathrm{x}$ & $\mathrm{x}$ & 73 & $\mathrm{c} 1$ & $\mathrm{npk}$ & 3 \\
\hline & L. stichae Harring, 1913 & $\mathrm{x}$ & $\mathrm{x}$ & 92 & $\mathrm{c} 1$ & npk & 3 \\
\hline & L. undulata Hauer, 1938 & & $\mathrm{x}$ & 48 & $\mathrm{c} 1$ & $\mathrm{npk}$ & 3 \\
\hline & L. papuana (Murray, 1913) & $\mathrm{x}$ & $\mathrm{x}$ & 120 & $\mathrm{c} 1$ & $\mathrm{npk}$ & 3 \\
\hline & L. pyriformis (Daday, 1905) & $\mathrm{x}$ & $\mathrm{x}$ & 67 & $\mathrm{c} 1$ & npk & 3 \\
\hline \multirow[t]{4}{*}{ Conochilidae } & Conochilus coenobasis (Skorikov, 1914) & $\mathrm{x}$ & $\mathrm{x}$ & 250 & $\mathrm{c} 2$ & $\mathrm{npk}$ & 1 \\
\hline & C. dossuaris Hudson, 1885 & $\mathrm{x}$ & $\mathrm{x}$ & 500 & $\mathrm{c} 2$ & $\mathrm{pk}$ & 1 \\
\hline & C. natans (Seligo, 1900) & $\mathrm{x}$ & $\mathrm{x}$ & 510 & c2 & $\mathrm{pk}$ & 1 \\
\hline & C. unicornis (Rousselet, 1892) & $\mathrm{x}$ & $\mathrm{x}$ & 510 & $\mathrm{c} 2$ & $\mathrm{npk}$ & 1 \\
\hline \multirow[t]{10}{*}{ Colurellidae } & Colurella obtusa (Gosse, 1886) & $\mathrm{x}$ & $\mathrm{x}$ & 60 & $\mathrm{c} 1$ & $\mathrm{npk}$ & 1 \\
\hline & Colurella sp. & $\mathrm{x}$ & $\mathrm{x}$ & & & & \\
\hline & Elosa sp. & $\mathrm{x}$ & $\mathrm{x}$ & & & & \\
\hline & Lepadella patella (O. F. Müller, 1786) & $\mathrm{x}$ & $\mathrm{x}$ & 145 & $\mathrm{c} 1$ & $\mathrm{npk}$ & 1 \\
\hline & L. benjamini Harring, 1916 & $\mathrm{x}$ & & 114 & $\mathrm{c} 1$ & $\mathrm{npk}$ & 1 \\
\hline & L. brasiliensis Koste, 1972 & $\mathrm{x}$ & $\mathrm{x}$ & 112 & $\mathrm{c} 1$ & npk & 1 \\
\hline & L. donneri Koste, 1972 & $\mathrm{x}$ & $\mathrm{x}$ & 140 & $\mathrm{c} 1$ & $\mathrm{npk}$ & 1 \\
\hline & L. quadricarinata (Stenroos, 1898) & $\mathrm{x}$ & $\mathrm{x}$ & 92 & $\mathrm{c} 1$ & $\mathrm{npk}$ & 1 \\
\hline & L. rhomboides (Gosse, 1886) & $\mathrm{x}$ & & 120 & $\mathrm{c} 1$ & npk & 1 \\
\hline & L. ovalis (O. F. Müller, 1786) & $\mathrm{x}$ & $\mathrm{x}$ & 170 & $\mathrm{c} 1$ & $\mathrm{npk}$ & 1 \\
\hline \multirow[t]{2}{*}{ Dicranophoridae } & Dicranophorus cf caudatus (Ehrenberg, 1834) & $\mathrm{x}$ & $\mathrm{x}$ & 310 & $\mathrm{c} 2$ & $\mathrm{pk}$ & 1 \\
\hline & Dicranophorus sp. & $\mathrm{x}$ & $\mathrm{x}$ & & & & \\
\hline \multirow[t]{5}{*}{ Euchlanidae } & Dipleuchlanis p. propatula (Gosse, 1886$)$ & $\mathrm{x}$ & $\mathrm{x}$ & 508 & $\mathrm{c} 2$ & $\mathrm{npk}$ & 1 \\
\hline & D.p. f. macrodactyla (Hauer, 1965) & $\mathrm{x}$ & $\mathrm{x}$ & 244 & $\mathrm{c} 2$ & npk & 1 \\
\hline & Euchlanis dilatata Ehrenberg, 1832 & $\mathrm{x}$ & $\mathrm{x}$ & 320 & $\mathrm{c} 2$ & npk & 1 \\
\hline & E. dilatata cf luckisiana (Hauer, 1930) & $\mathrm{x}$ & $\mathrm{x}$ & 315 & $\mathrm{c} 2$ & npk & 1 \\
\hline & E. incisa Carlin, 1939 & & $\mathrm{x}$ & 270 & $\mathrm{c} 2$ & $\mathrm{npk}$ & 1 \\
\hline
\end{tabular}




\begin{tabular}{|c|c|c|c|c|c|c|c|}
\hline \multirow[t]{2}{*}{ Families } & \multirow[t]{2}{*}{ Taxa } & \multicolumn{2}{|c|}{ Occurrence } & \multirow{2}{*}{$\mathbf{t}(\mu \mathbf{m})$} & \multirow[t]{2}{*}{ cl } & \multirow[t]{2}{*}{ h } & \multirow[t]{2}{*}{ b } \\
\hline & & $\mathbf{L}$ & $\mathbf{R}$ & & & & \\
\hline & Manfredium eudactilota Gallagher, 1957 & $\mathrm{x}$ & $\mathrm{x}$ & 760 & c3 & npk & 1 \\
\hline \multirow[t]{5}{*}{ Filinidae } & Filinia longiseta (Ehrenberg, 1834) & $\mathrm{x}$ & $\mathrm{x}$ & 250 & $\mathrm{c} 2$ & $\mathrm{pk}$ & 1 \\
\hline & F. cf Terminalis (Plate, 1886) & $\mathrm{x}$ & $\mathrm{x}$ & 180 & $\mathrm{c} 1$ & $\mathrm{pk}$ & 1 \\
\hline & F. opoliensis (Zacharias, 1891) & $\mathrm{x}$ & $\mathrm{x}$ & 204 & $\mathrm{c} 2$ & $\mathrm{pk}$ & 1 \\
\hline & F. pjeleri Hutchinson, 1964 & $\mathrm{x}$ & $\mathrm{x}$ & 200 & $\mathrm{c} 2$ & $\mathrm{pk}$ & 1 \\
\hline & F.saltator (Gosse, 1886) & $\mathrm{x}$ & $\mathrm{x}$ & 135 & $\mathrm{c} 1$ & $\mathrm{pk}$ & 1 \\
\hline Asplanchinidae & Asplanchna sieboldi (Leydig, 1854) & $\mathrm{x}$ & $\mathrm{x}$ & 2500 & $\mathrm{c} 4$ & $\mathrm{pk}$ & 1 \\
\hline Proalidae & Proales cf decipiens (Ehrenberg, 1831) & & $\mathrm{x}$ & 180 & $\mathrm{c} 1$ & $\mathrm{npk}$ & 1 \\
\hline \multirow[t]{5}{*}{ Flosculariidae } & Floscularia cf ringens (Linné, 1758) & $\mathrm{x}$ & $\mathrm{x}$ & 1900 & $\mathrm{c} 4$ & $\mathrm{pk}$ & 1 \\
\hline & Floscularia sp. & $\mathrm{x}$ & $\mathrm{x}$ & & & & \\
\hline & Pompholyx cf triloba Pejler, 1957 & $\mathrm{x}$ & $\mathrm{x}$ & 120 & $\mathrm{c} 1$ & npk & 1 \\
\hline & Ptygura cf longicornis Donner, 1954 & $\mathrm{x}$ & $\mathrm{x}$ & 350 & $\mathrm{c} 2$ & npk & 1 \\
\hline & Ptygura sp. & $\mathrm{x}$ & $\mathrm{x}$ & & & & \\
\hline \multirow[t]{5}{*}{ Gastropodidae } & Ascomorpha ecaudis (Perty, 1859) & $\mathrm{x}$ & $\mathrm{x}$ & 200 & c2 & npk & 1 \\
\hline & A. saltans Bartsch, 1870 & $\mathrm{x}$ & $\mathrm{x}$ & 165 & $\mathrm{c} 1$ & $\mathrm{pk}$ & 1 \\
\hline & A. ovalis (Bergendahl, 1892) & & $\mathrm{x}$ & 200 & $\mathrm{c} 2$ & npk & 1 \\
\hline & A. klementi Hauer, 1972 & $\mathrm{x}$ & $\mathrm{x}$ & 200 & c2 & $\mathrm{pk}$ & 1 \\
\hline & Gastropus sp. & $\mathrm{x}$ & & & & & \\
\hline \multirow[t]{2}{*}{ Hexarthridae } & Hexarthra mira (Hudson, 1871) & $\mathrm{x}$ & $\mathrm{x}$ & 400 & $\mathrm{c} 2$ & $\mathrm{pk}$ & 1 \\
\hline & H. intermedia Wiszniewski, 1929 & $\mathrm{x}$ & $\mathrm{x}$ & 234 & $\mathrm{c} 2$ & $\mathrm{pk}$ & 1 \\
\hline \multirow[t]{2}{*}{ Collothecidae } & Collotheca $\mathrm{cf}$ ornata (Ehrenberg, 1832) & $\mathrm{x}$ & & 1200 & $\mathrm{c} 4$ & $\mathrm{npk}$ & 1 \\
\hline & Collotheca sp. & $\mathrm{x}$ & & & & & \\
\hline \multirow[t]{5}{*}{ Mytilinidae } & Mytilinia bicarinata (Perty, 1850) & $\mathrm{x}$ & $\mathrm{x}$ & 185 & $\mathrm{c} 1$ & npk & 1 \\
\hline & M. bisulcata (Lucks, 1912) & $\mathrm{x}$ & $\mathrm{x}$ & 180 & $\mathrm{c} 1$ & npk & 1 \\
\hline & M. macrocera (Jennings, 1894) & $\mathrm{x}$ & & 320 & $\mathrm{c} 2$ & $\mathrm{pk}$ & 1 \\
\hline & M. trigona (Gosse, 1886) & $\mathrm{x}$ & & 253 & $\mathrm{c} 2$ & $\mathrm{pk}$ & 1 \\
\hline & M. ventralis (Ehrenberg, 1832) & $\mathrm{x}$ & $\mathrm{x}$ & 380 & $\mathrm{c} 2$ & $\mathrm{npk}$ & 1 \\
\hline \multirow[t]{7}{*}{ Notommatidae } & Cephalodella sterea (Gosse, 1887) & $\mathrm{x}$ & $\mathrm{x}$ & 250 & $\mathrm{c} 2$ & $\mathrm{pk}$ & 1 \\
\hline & Cephalodella sp. & $\mathrm{x}$ & $\mathrm{x}$ & & & & \\
\hline & Monomata cf maculata Harring \& Myers, 1924 & $\mathrm{x}$ & $\mathrm{x}$ & 680 & $\mathrm{c} 3$ & $\mathrm{npk}$ & 1 \\
\hline & Monommata sp. & $\mathrm{x}$ & $\mathrm{x}$ & & & & \\
\hline & Notommata copeus Ehrenberg, 1834 & $\mathrm{x}$ & $\mathrm{x}$ & 1100 & $\mathrm{c} 4$ & npk & 1 \\
\hline & Notommata sp. & $\mathrm{x}$ & $\mathrm{x}$ & & & & \\
\hline & Scaridium sp. & $\mathrm{x}$ & $\mathrm{x}$ & & & & \\
\hline \multirow[t]{6}{*}{ Synchaetidae } & Polyarthra dolicoptera Idelson, 1925 & $\mathrm{x}$ & $\mathrm{x}$ & 140 & $\mathrm{c} 1$ & $\mathrm{pk}$ & 1 \\
\hline & P. remata (Skorikov, 1896$)$ & $\mathrm{x}$ & $\mathrm{x}$ & 120 & $\mathrm{c} 1$ & $\mathrm{npk}$ & 1 \\
\hline & P. vulgaris Carlin, 1943 & $\mathrm{x}$ & $\mathrm{x}$ & 145 & $\mathrm{c} 1$ & $\mathrm{pk}$ & 1 \\
\hline & Ploesoma truncata (Levander, 1894) & $\mathrm{x}$ & $\mathrm{x}$ & 300 & $\mathrm{c} 2$ & $\mathrm{pk}$ & 1 \\
\hline & Synchaeta longipes Gosse, 1887 & $\mathrm{x}$ & $\mathrm{x}$ & 204 & $\mathrm{c} 2$ & $\mathrm{pk}$ & 1 \\
\hline & S. stylata Wiezejski, 1893 & $\mathrm{x}$ & $\mathrm{x}$ & 313 & $\mathrm{c} 2$ & $\mathrm{pk}$ & 1 \\
\hline \multirow[t]{5}{*}{ Testudinellidae } & Testudinella ahlstromi (Hauer, 1956) & & $\mathrm{x}$ & 122 & $\mathrm{c} 1$ & $\mathrm{npk}$ & 1 \\
\hline & T. mисronata (Gosse, 1886) & $\mathrm{x}$ & $\mathrm{x}$ & 500 & $\mathrm{c} 2$ & $\mathrm{npk}$ & 1 \\
\hline & T. ohlei Koste, 1972 & & $\mathrm{x}$ & 140 & $\mathrm{c} 1$ & $\mathrm{npk}$ & 1 \\
\hline & T. tridentata Smirnov, 1931 & $\mathrm{x}$ & $\mathrm{x}$ & 185 & $\mathrm{c} 1$ & $\mathrm{npk}$ & 1 \\
\hline & T. patina $($ Hermann, 1783) & $\mathrm{x}$ & $\mathrm{x}$ & 350 & $\mathrm{c} 2$ & $\mathrm{npk}$ & 1 \\
\hline \multirow[t]{3}{*}{ Trichotridae } & Macrochaetus collinsis (Gosse, 1867) & & $\mathrm{x}$ & 250 & $\mathrm{c} 2$ & $\mathrm{npk}$ & 1 \\
\hline & M. sericus (Thorpe, 1893) & & $\mathrm{x}$ & 160 & c1 & npk & 1 \\
\hline & Tricothria tetractis (Ehrenberg, 1830) & $\mathrm{x}$ & $\mathrm{x}$ & 295 & $\mathrm{c} 2$ & $\mathrm{npk}$ & 1 \\
\hline
\end{tabular}


Species richness was higher in the river than the lake, mainly during high water, because of the higher number of non-planktonic taxa in the river. This number increased in the high water period because of (i) washing of littoral vegetation, with consequent increases in the number of taxa from benthic and periphyton communities entering open water; and (ii) the contribution from species entering from other environments which are isolated during other times of year. More planktonic taxa were recorded during low water in both river and lake.

The faunistic similarity obtained in the environemnts showed that in the high water period the contact of the lake and the river was intensified if more water was present. This fact was observed for the rotifers and microcrustaceans fauna (cladocerans and copepods) (Bonecker etal., 1994; Lima et al., 1996) in the same environments. Thomaz et al. (1997) showed that during this period a greater environmental homogenity was observed, which was mainly due to the mixed water.

Sharma \& Naik (1996) discussed variations in the composition of the non-planktonic community over spatial scales, and noted that they were influenced by other factors such as marginal and submarginal aquatic vegetation. They considered that rotifer richness in the river increased because of the contribution from the fauna upstream. In Venezuelan rivers, Zoppi de Roa et al. (1990) recorded more benthic and periphytic species in the zooplankton, mainly species of Lecane, which was associated with aquatic vegetation

More planktonic taxa were recorded in the river because of, among other factors, the low current flow $(0.11-0.50 \mathrm{~m} / \mathrm{s})$. According to Paggi \& José de Paggi (1990), low current and shallow depths such as in the Paraná (1.6-3.4 m), were important factors in increasing richness of rotifers.

Pjeler \& Bérzins (1989) suggested that some Brachionidae, such as Brachionus angularis and Keratella cochlearis, were both planktonic and non-planktonic, while others such as Anueropsis fissa, Platyias quadricornis, Plationus patulus, and B. quadridentatus were planktonic. All these taxa were found in the river and the lake, except for A. fissa which occurred only in the lake.

Several studies have demonstrated the occurrence of non-planktonic taxa in open waters, because of increased fluviometric levels and the presence of littoral vegetation (Shiel et al., 1986; José de Paggi, 1993). Our results showed that non-planktonic and planktonic taxa could probably establish themselves in open water during both low and high water periods. However, increase in the fluviometric level also contributed to increased richness, and no planktonic taxon predominated during high water.

The establishment of both non-planktonic and planktonic taxa in open water of the small lakes with marginal vegetation indicated that different niches were being occupied. This occurs because of (i) differences in particulate food resources, for example Asplanchna feeds on larger particle sizes than do Brachionus and Trichocerca, or (ii) environmental characteristics; for instance, Anueropsis, Collotheca, and Horäella were recorded in the lake, and Trochocosphaera and Proales in the river.

Interactions among each other due to different relationships between each other and the environment and the species coexisted. Interspecific interactions were related to competition and predation, and the relationships of species with their environment depended on the limnological characteristics and their seasonal variations. Many studies have shown the importance of environmental conditions for the distribution of species in the environment, as well as for the coexistence of a large number of rotifer taxa in the plankton (Green, 1972; Bonecker, 1994; Bozelli, 1994). According to Pjeler \& Berzins (1995) most rotifer taxa were not restricted to a single habitat type.

The highest number of taxa was recorded in the size classes up to $600 \mu \mathrm{m}$ (c1 and c2). That size is characteristic of the group, most of which are between 100 and $500 \mu \mathrm{m}$ long (Koste, 1978). The smaller taxa predominated in the high water period. Papinska (1990) showed that the higher number of rotifer taxa of different sizes in the Vistula River (Poland) might be related to the 
amount of available food, i.e., in conditions of low concentrations of food, more smaller species might occur. However, our results suggested that other factors might be related to the size of the predominant rotifer taxa, because in the hydrological period (high water) when a low number of smaller taxa occurred, the chlorophyll-a concentration was lower (Bonecker, 1998).

Another factor that could explain the occurrence of the highest number of small taxa during the high water period was predation on the larger taxa, because more larval fish (Baumgartner et al., 1997) and Chaoborus (Diptera-Insecta) (Takeda et al., 1991) occurred at that time, mainly in the lake.

\section{ACKNOWLEDGEMENTS}

We would like to thank Drs. Reinaldo Luiz Bozelli, Susana José de Paggi and Elsa Rodrigues Hardy for suggestions, and CNPq and DBI/Nupelia for financial and logistical support. Dr. Janet W. Reid revised the English text.

\section{RESUMO}

A composição e ocorrência dos rotíferos foi investigada during dois períodos hidrológicos distintos em dois ambientes (rio e lagoa). As coletas foram realizadas diariamente (14 dias) à superfície na região pelágica de cada ambiente. A similaridade faunística entre os ambientes foi avaliada através do índice de Jaccard. Foram identificados 143 táxons. O maior número de táxons foi registrado no rio. A similaridade faunística entre os ambientes foi maior no período de águas altas. Os táxons planctônicos predominaram, sendo que a diferença para os não planctônicos não foi expressiva. No rio, destacaram-se esses últimos táxons, enquanto que na lagoa prevaleceram tanto os táxons planctônicos como os não planctônicos. Verificou-se que ocorreu um maior número de táxons de menor tamanho ( $\geq 200 \mu \mathrm{m}$ a $600 \mu \mathrm{m})$ nos dois ambientes. Esse fato está relacionado com a pequena profundidade dos ambientes e a velocidade de corrente no rio, o que permite o deslocamento dos táxons bentônicos e perifíticos do fundo e da região litorânea para a coluna de água.

\section{REFERENCES}

Baumgartner, G.; Nakatani, K.; Cavicchioli, M. \& Baumgartner, M.S.T. (1997), Some aspects of the ecology of fish larvae in the floodplain of the high Paraná river, Brazil. Rev. Brasil. Zool., 14 (3), 551-563.

Bonecker, C.C. (1994), Variações espaçotemporais da fauna de rotíferos em distritos ambientes da planície de inundação do alto rio Paraná (MS). Dissertation, State University of Maringá, Maringá, Paraná 25 p.

Bonecker, C.C. \& Lansac-Tôha, F.A. (1996), Community structure of rotifers in two environments of the high River Paraná floodplain (MS), Brazil. Hydrobiologia, 325, 137-150.

Bonecker, C.C. (1998), Estudos Populacionais dos rotíferos em diferentes ambientes da planície de inundação do alto rio Paraná-MS. Thesis State University of Maringá, Maringá, Paraná 75 p.

Bozelli, R.L. (1992), Zooplankton comunity of Batata and Mussurá Lakes and of the Trombetas River, state of Pará, Brazil. Amazoniana, 12 (2), 239-261.

Bozelli, R.L. (1994), Zooplankton comunity density in relation to water level fluctuation and inorganic turbity in an Amazonian lake, "Lago Batata", State of Pará, Brazil. Amazoniana, 13 (1/2), 17-32.

Espindola, E.G.; Matsumura-Tundisi, T. \& Moreno, I.H. (1996), Efeitos da dinâmica hidrobiológica do sistema Pantanal matogrossense sobre a estrutura da comunidade de zooplâncton da lagoa Albuquerque. Acta Limnol. Brasil., 8, 37-57.

Green, J. (1972), Freshwater ecology in the Mato Grosso, Central Brazil. III. Associations of Rotifera in meander lakes of the rio Suiá Missú. Hid. Nat. Hist., 6, 220241.

Hardy, E.R. (1980), Composição do zooplâncton em cinco lagos da Amazônia Central. Acta Amazonica, 10 (3), 577-609.

Hilmann, T.J. \& Shiel, R.J. (1991), Macro-and microinvertebrates in Australian billabongs. Verh. Internat. Verein. Limnol., 24, 15811587. 
José de Paggi, S. (1989), Rotíferos de algunas provincias del noroeste argentino. Rev. Hydrobiol. Trop., 22 (3), 223-238.

José de Paggi, S. (1993), Composition and seasonality of planktonic rotifers in limnetic and littoral regions of a floodplain lake (Paraná river system). Hydrobiol. trop., 26 (1), 53-63.

José de Paggi, S. (1996), Rotifera (Monogononta) diversity in subtropical waters of Argentina. Annls Limnol., 32 (4), 209-220.

Krebs, C. J. (1989), Ecological Methodology. Harper and Row, Publishers, New York, $654 \mathrm{p}$.

Koste, W. (1978), Rotatoria Die Rodertiere Mitteleuropas begründet von Max Voigt Monogononta. 2. Auflage neubearbeitet von Walter Koste. Gebrüder Borntraeger. Berlin. 1 (673p); 2 (238 p).

Koste, W. \& Robertson, B. (1983), Taxonomic studies of the Rotifera (Phylum Aschelminthes) from a Central Amazonian varzea lake, Lago Camaleão (Ilha de Marchantaria, Rio Solimões, Amazonas, Brazil). Amazoniana, 8 (2), 225-254.

Koste, W. \& Shiel, R.J. (1986), Rotifera from Australian inland waters. I. Bdelloidea (Rotifera: Digononta). Aust. J. Mar. Freshw. Res., 37, 765-792.

Lansac-Tôha, F.A.; Bonecker, C.C.; Velho, L.F.M. \& Lima, A.F. (1997), Comunidade zooplanctônica. In- A Planície de Inundação do Alto Rio Paraná: aspectos físicos, químicos, biológicos e socioeconômicos, ed. Vazzoler, A.E.A.M.; Agostinho, A.A.; Hahn, N.S. Editora da Universidade Estadual de Maringá, Maringá, pp. 117-155.

Lima, AF.; Lansac-Tôha, F.A \& Bonecker, C.C. (1996), Zooplankton in the floodplain of a tributary to the Paraná river in Mato Grosso do Sul, Brazil. Stud Neotrop Fauna \& Environ., 31 (2), 112-116.

Lopes, C. \& Ochoa E. (1995), Rotíferos (Monogononta) de la cuenca Río GuasareLimón, Venezuela. Biol. Trop., 43 (1-3), 189193.

Papinski, K. (1990), Abundance and composition of rotifers in the Vistula River. Pol. Arch. Hydrobiol., 37 (3), 449-459.

Paggi, J.C. \& José De Paggi, S. (1990), Zooplâncton de ambientes lóticos e lênticos do rio Paraná médio. Acta Limnol. Brasil., 3 , 685-719.

Pjeler, B. \& Berzins, B. (1989), On choice of substrate and habitat in brachionid rotifers. Hydrobiologia, 186/187, 137-144.

Pjeler, B. \& Berzins, B. (1995), Relation to habitat in rotifers. Hydrobiologia, 313/314, 267-278.

Robertson, B.A. \& Hardy, E.R. (1984), Zooplankton of Amazonian lakes and rivers. In- The Amazon Sioli, H. ed,. Monogr. Biol, 56, 337-352.

Rocha, O; Sendacz, S. \& Matsumura-Tundisi, T. (1995), Composition, biomass and productivity of zooplankton in natural lakes and reservoirs of Brazil. In- Limnology in Brazil, ed. Tundisi, J.G.; Bicudo, C.E.M.; Matsumura-Tundisi, T. Rio de Janeiro, ABC/SBL., pp. 151-165.

Rossa, D.C. (1997), Composição e abundância do zooplâncton da região litorânea de uma lagoa de várzea e um rio da planície de inundação do alto rio Paraná - MS. Monograph., State University of Maringá, Maringá. Paraná. 39p.

Segers, H.; Nwadiaro, C.S. \& Dumont, H.J. (1993), Rotifera of some lakes in the floodplain of the River Niger (Imo State, Nigeria). Hydrobiologia, 250, 63-79.

Segers, H. (1995), Rotifera. The Lecanidae (Monogononta). The Hague, The Netherlands: SPB Academics. Guides to the identification of the microinvertebrates of the continental waters of the world, 2, 226p.

Sendacz, S. (1997), Zooplankton studies of floodplain lakes of the Upper Paraná River, São Paulo State, Brazil. Verh. Internat. Verein. Limnol., 26, 621-627.

Serafim, M.Jr (1997), Heterogeneidade espacial e temporal da comunidade zooplanctônica do sistema rio Ivinhema-lagoa dos Patos, Planície de inundação do alto rio Paraná (MS). Dissertation, State University of Maringá, Maringá, Paraná. 44 p.

Sharma, B.K. \& Naik, L.P. (1996), Results on planktonic rotifers in the Narmada River (Madhya Pradesh, Central India). Tropical Limnology, 189-198.

Shiel, R. J. (1986), Zooplankton of the MurrayDarling system. In- The Ecology of River Systems, ed. B.R. Davies \& K.F. Walker. Monogr. Biol., 60, 661-677. 
Souza, M. C.; Cislinksi, J. \& Romagnolo, M. (1997), Levantamento florístico. In- $A$ Planície de Inundação do Alto Rio Paraná: aspectos físicos, químicos, biológicos e socioeconômicos, ed. Vazzoler, A.E.A.M.; Agostinho, A.A.; Hahn, N.S. Editora da Universidade Estadual de Maringá, Maringá, pp. 343-368.

Takeda, A.M.; Shimizu, G.Y.; Shulz, G.M. \& Silva, A.C.M. (1991), Zoobentos de quatro lagoas de várzea do alto rio Paraná (MSBrasil). Influência do regime hidrológico sobre a comunidade. Revista UNIMAR, 13 (2), 365-387.

Tan, L.W. \& Shiel, R.J. (1993), Responses of billabong rotifer communities to inundation. Hydrobiologia, 225/226, 361-369.

Thomaz, S.M.; Roberto, M.C.; Lansac-Tôha, F.A.; Esteves, F.A. \& Lima, A.F. (1991), Dinâmica temporal dos principais fatores limnológicos do rio Baía-planície de inundação do alto rio Paraná-MS, Brasil. Revista UNIMAR, 13 (2), 299-312.
Seasonal variation of some limnological factors of a high Paraná river várzea lake Guaraná Lake - State of Mato Grosso do Sul - Brazil. Rev. Hydrobiol. Trop., 25 (4), 269276.

Twombly, S. \& Lewis Jr., W.M. (1987), Zooplankton abundance and species composition in Laguna la Orsinera, a Venezuelan floodplain lake. Arch. Hydrobiol. Suppl., 79 (1), 87-107.

Vásquez, E. (1984), El zooplancton de la seccion baja de un rio de aguas negras (rio Caroni) y de un embalse hidroelectrico (Macagua I), Venezuela. Mem. Soc. Cienc. Nat. La Salle, 41, 109-130.

Vásquez, E. \& Rey, J. (1989), A longitudional study of zooplankton along the lower Orinoco River and its Delta (Venezuela). Annls. Limnol., 28 (1), 3-18.

Zoppi De Roa, E.;Vásquez, W.; Colomine, G. \& Pardo, M.J. (1990), Composicion preliminar del zooplancton del rio Churun (Auyantepuy, Venezuela). Soc. Cienc. Nat. La Salle, 41, 29-45. 